\title{
Integral operators with rough kernels in variable Lebesgue spaces
}

\author{
Marta Urciuolo - Lucas Vallejos
}

February 19, 2020

\begin{abstract}
In this paper we study integral operators with kernels

$K(x, y)=k_{1}\left(x-A_{1} y\right) \ldots k_{m}\left(x-A_{m} y\right)$,

$k_{i}(x)=\frac{\Omega_{i}(x)}{|x|^{n / q_{i}}}$ where $\Omega_{i}: \mathbb{R}^{n} \rightarrow \mathbb{R}$ are homogeneous functions of degree zero, satisfying a size and a Dini condition, $A_{i}$ are certain invertible matrices, and $\frac{n}{q_{1}}+\ldots \frac{n}{q_{m}}=n-\alpha, 0 \leq \alpha<n$.

We obtain the boundedness of this operator from $L^{p(\cdot)}$ into $L^{q(\cdot)}$ for $\frac{1}{q(\cdot)}=\frac{1}{p(\cdot)}-\frac{\alpha}{n}$, for certain exponent functions $p$ satisfying weaker conditions than the classical log-Hölder conditions.

123
\end{abstract}

\section{Introduction}

Given a measurable set $\Omega \subseteq \mathbb{R}^{n}$ we denote with $\mathcal{P}(\Omega)$ the family of measurable function $p(\cdot): \Omega \rightarrow[1, \infty]$. Given $p(\cdot) \in \mathcal{P}(\Omega)$ let $L^{p(\cdot)}(\Omega)$ be the Banach space of measurable functions $f$ on $\Omega$ such that for some $\lambda>0$,

$$
\rho_{p(\cdot), \Omega}(f / \lambda)<\infty
$$

where

$$
\rho_{p(\cdot), \Omega}(f)=\int_{\Omega \backslash \Omega_{\infty}}|f(x)|^{p(x)} d x+\|f\|_{L^{\infty}\left(\Omega_{\infty}\right)},
$$

$\Omega_{\infty}=\{x \in \Omega: p(x)=\infty\}$, with norm

$$
\|f\|_{L^{p(\cdot)}(\Omega)}=\inf \left\{\lambda>0: \rho_{p(\cdot), \Omega}(f / \lambda) \leq 1\right\} .
$$

We will denote $\|f\|_{p(\cdot)}$ instead of $\|f\|_{L^{p(\cdot)}(\Omega)}$ if the role of the set $\Omega$ is clear enough. These spaces are known as variable exponent spaces and are a generalization of the classical Lebesgue spaces $L^{p}\left(\mathbb{R}^{n}\right)$. They have been widely studied lately. See for example [1], [3] and [4]. The first step was to determine sufficient conditions on $p(\cdot)$ for the boundedness on $L^{p(\cdot)}$ of the Hardy Littlewood maximal operator

$$
\mathcal{M} f(x)=\sup _{B} \frac{1}{|B|} \int_{B}|f(y)| d y,
$$

\footnotetext{
${ }^{1}$ Partially supported by CONICET and SECYTUNC

${ }^{2}$ Math. subject classification: 42B25, 42B35.

${ }^{3}$ Key words: Variable Exponents, Fractional Integrals.
} 
where the supremun is taken over all balls $B$ containing $x$. Analogously for $0<\alpha<n$ we recall the definition of the fractional maximal operator

$$
M_{\alpha} f(x)=\sup _{Q \ni x} \frac{1}{|B|^{1-\frac{\alpha}{n}}} \int_{B}|f(y)| d y,
$$

where also the supremun is taken over all balls $B$ containing $x$.

We also define $M_{\alpha, s}$, for all $1 \leq s<\infty$,

$$
M_{\alpha, s} f=\left(M_{\alpha . s}|f|^{s}\right)^{1 / s} .
$$

Let $p_{-}=e s s \inf p(x)$ and let $p_{+}=e s s \sup p(x)$.

Definition 1 Given $\Omega \subseteq \mathbb{R}^{n}$, and function $r(\cdot): \Omega \rightarrow \mathbb{R}$, we say that $r(\cdot)$ is locally log-Hölder continuous, and denote this by $r(\cdot) \in L H_{0}(\Omega)$, if there exist a constant $C_{0}$ such that for all $x, y \in \Omega,|x-y|<\frac{1}{2}$,

$$
|r(x)-r(y)| \leq \frac{C_{0}}{-\log (|x-y|)} .
$$

We say that $r(\cdot)$ is log-Hölder continuous at infinity, and denote this by $r(\cdot) \in$ $L H_{\infty}(\Omega)$, if there exist constants $C_{\infty}$ and $r^{\infty}$ such that for all $x \in \Omega$,

$$
\left|r(x)-r^{\infty}\right| \leq \frac{C_{\infty}}{\log (e+|x|)} .
$$

In [3] , D. Cruz Uribe, A. Fiorenza and C. J. Neugebauer proved the following result. If $p(\cdot) \in \mathcal{P}\left(\mathbb{R}^{n}\right), 1<p_{-} \leq p_{+}<\infty$ and $p(\cdot) \in L H_{0}\left(\mathbb{R}^{n}\right) \cap L H_{\infty}\left(\mathbb{R}^{n}\right)$, then the Hardy Littlewood maximal operator is bounded on $L^{p(\cdot)}\left(\mathbb{R}^{n}\right)$. In [2] (chapter 4) the authors show that the boundedness of the maximal operator can be obtained under weaker conditions on the exponent $p(\cdot)$. They define the $N_{\infty}-$ condition as follows,

Definition 2 Given $\Omega \subseteq \mathbb{R}^{n}$ and $p(\cdot) \in \mathcal{P}\left(\mathbb{R}^{n}\right)$, we say that $p(\cdot) \in N_{\infty}(\Omega)$ if there exist constants $\Lambda_{\infty}$ and $p_{\infty}$ such that

$$
\int_{\Omega_{+}} \exp \left(-\Lambda_{\infty}\left|\frac{1}{p(x)}-\frac{1}{p_{\infty}}\right|^{-1}\right) d x<\infty .
$$

where $\Omega_{+}=\left\{x \in \Omega:\left|\frac{1}{p(x)}-\frac{1}{p_{\infty}}\right|>0\right\}$.

Also, in [2], the authors define the $K_{0}-$ condition as follows,

Definition 3 Given $p(\cdot) \in \mathcal{P}\left(\mathbb{R}^{n}\right)$, then $p(\cdot) \in K_{0}\left(\mathbb{R}^{n}\right)$ if there exists a constant $C$ such that, for every cube $Q$,

$$
\left\|\chi_{Q}\right\|_{L^{p(\cdot)\left(\mathbb{R}^{n}\right)}}\left\|\chi_{Q}\right\|_{L^{p^{(\cdot)}\left(\mathbb{R}^{n}\right)}} \leq C|Q|
$$

They prove the following result. If $p(\cdot) \in \mathcal{P}\left(\mathbb{R}^{n}\right), 1<p_{-} \leq p_{+}<\infty$ and $p(\cdot) \in K_{0}\left(\mathbb{R}^{n}\right) \cap N_{\infty}\left(\mathbb{R}^{n}\right)$, then the Hardy Littlewood maximal operator is bounded on $L^{p(.)}\left(\mathbb{R}^{n}\right)$. They also show that $L H_{0}\left(\mathbb{R}^{n}\right) \cap L H_{\infty}\left(\mathbb{R}^{n}\right) \subset$ $K_{0}\left(\mathbb{R}^{n}\right) \cap N_{\infty}\left(\mathbb{R}^{n}\right)$ and they give an example that shows that the the inclusion 
is strict.

Let $0 \leq \alpha<n, m \in \mathbb{N}$. For $1 \leq i \leq m$, let $1<q_{i}<\infty$ such that $\frac{n}{q_{1}}+\cdots+\frac{n}{q_{m}}=n-\alpha$. For $\alpha=0$ we take $m>1$. We denote by $\Sigma=\Sigma_{n-1}$ the unit sphere in $\mathbb{R}^{n}$. Let $\Omega_{i} \in L^{1}(\Sigma)$. If $x \neq 0$, we write $x^{\prime}=x /|x|$. We extend this function to $\mathbb{R}^{n} \backslash\{0\}$ as $\Omega_{i}(x)=\Omega_{i}\left(x^{\prime}\right)$.

Let

$$
k_{i}(x)=\frac{\Omega_{i}(x)}{|x|^{n / q_{i}}},
$$

and let

$$
T_{\alpha} f(x)=\int_{\mathbb{R}^{n}} K(x, y) f(y) d y,
$$

with $K(x, y)=k_{1}\left(x-A_{1} y\right) \ldots k_{m}\left(x-A_{m} y\right)$, where $A_{i}$, are certain invertible matrices and $f \in L_{\text {loc }}^{\infty}\left(\mathbb{R}^{n}\right)$. In [6] the authors consider the operator $T_{\alpha}$ defined in (3) where, for $1 \leq i \leq m, k_{i}$ is given by (2). For $1 \leq p \leq \infty$ and $\Omega_{i} \in L^{1}(\Sigma)$, they define the $L^{p}$ - modulus of continuity as

$$
\varpi_{i, p}(t)=\sup _{|y| \leq t}\left\|\Omega_{i}(\cdot+y)-\Omega_{i}(\cdot)\right\|_{p, \Sigma} .
$$

They make the following hypothesis about the functions $\Omega_{i}, 1 \leq i \leq m$,

$\left(H_{1}\right)$ There exists $p_{i}>q_{i}$ such that $\Omega_{i} \in L^{p_{i}}(\Sigma)$,

$$
\left(H_{2}\right) \int_{0}^{1} \varpi_{i, p_{i}}(t) \frac{d t}{t}<\infty .
$$

They obtain the boundedness of this kind of operators in weighted Lebesgue spaces. We recall that a weight $\omega$ is a locally integrable and non negative function. The Muckenhoupt class $\mathcal{A}_{p}, 1<p<\infty$, is defined as the class of weights $\omega$ such that

$$
\sup _{Q}\left[\left(\frac{1}{|Q|} \int_{Q} \omega\right)\left(\frac{1}{|Q|} \int_{Q} \omega^{-\frac{1}{p-1}}\right)^{p-1}\right]<\infty,
$$

where $Q$ is a cube in $\mathbb{R}^{n}$.

For $p=1, \mathcal{A}_{1}$ is the class of weights $\omega$ satisfying that there exists $c>0$ such that

$$
\mathcal{M} \omega(x) \leq c \omega(x) \text { a.e. } x \in \mathbb{R}^{n} .
$$

We denote $[\omega]_{\mathcal{A}_{1}}$ the infimum of the constant $c$ such that $\omega$ satisfies the above inequation.

In this paper we study the boundedness of $T_{\alpha}$ on variable Lebesgue spaces. The exponent functions will satisfy certain regularity conditions and also certain relations with the different matrices $A_{i}$ involved in the kernel $K$. We will ask the hypothesis $p\left(A_{i} x\right) \leq p(x)$ a.e. $x \in \mathbb{R}^{n}$. In [9] we proved that this condition is in fact necessary in some particular cases. We will first prove the boundeness of the fractional maximal operator in variable Lebesgue spaces, with standard extrapolation techniques. Then we obtain the $L^{p(\cdot)}\left(\mathbb{R}^{n}\right)-L^{q(\cdot)}\left(\mathbb{R}^{n}\right)$ boundedess of $T_{\alpha}, \frac{1}{q(\cdot)}-\frac{1}{p(\cdot)}=\frac{\alpha}{n}$. To obtain this result we use the boundedness of the 
"sharp maximal function". We recall that given a function $f \in L_{l o c}^{1}\left(\mathbb{R}^{n}\right)$ we define the sharp maximal function

$$
M^{\#} f(x)=\sup _{B} \frac{1}{|B|} \int_{B}\left|f(y)-\frac{1}{|B|} \int_{B}\right| f|| d y,
$$

where $B$ is a ball containing $x$. In [2] the authors prove that given $p(\cdot)$ with $1 \leq p_{-} \leq p_{+}<\infty$, if the maximal operator is bounded on $L^{p^{\prime}(\cdot)}\left(\mathbb{R}^{n}\right)$ then there exists $c>0$ such that

$$
\sup _{t>0}\left\|t \chi_{\{x:|f(x)|>t\}}\right\|_{p(\cdot)} \leq c \sup _{t>0}\left\|t \chi_{\{x:|M \# f(x)|>t\}}\right\|_{p(\cdot)}
$$

and

$$
\|f\|_{p(\cdot)} \leq c\left\|M^{\#} f\right\|_{p(\cdot)} .
$$

\section{Main results}

In this paragraph we use the sharp maximal function to obtain the boundedness of the operator defined by (3).

In [5], B. Muckenhoupt y R.L. Wheeden define $\mathcal{A}(p, q), 1<p<\infty$ and $1<q<$ $\infty$, as the class of weights $\omega$ such that

$$
\sup _{Q}\left[\left(\frac{1}{|Q|} \int_{Q} \omega(x)^{q} d x\right)^{\frac{1}{q}}\left(\frac{1}{|Q|} \int_{Q} \omega(x)^{-p^{\prime}} d x\right)^{\frac{1}{p^{\prime}}}\right]<\infty .
$$

When $p=1, \omega \in \mathcal{A}(1, q)$ if only if

$$
\sup _{Q}\left[\left\|\omega^{-1} \chi_{Q}\right\|_{\infty}\left(\frac{1}{|Q|} \int_{Q} \omega(x)^{q} d x\right)^{\frac{1}{q}}\right]<\infty .
$$

They prove that $M_{\alpha}: L^{p}\left(\omega^{p}\right) \longrightarrow L^{q}\left(\omega^{q}\right)$ for weights $\omega \in \mathcal{A}(p, q), \frac{1}{p}-\frac{1}{q}=$ $\frac{\alpha}{n}$. They also obtain the corresponding weak type inequality. With classical extrapolation techniques we get the following result,

Lemma 1 Let $0<\alpha<n$ and let $p(\cdot) \in \mathcal{P}\left(\mathbb{R}^{n}\right)$, such that $1 \leq p_{-} \leq p_{+}<\frac{n}{\alpha}$. Let $q(\cdot) \in \mathcal{P}\left(\mathbb{R}^{n}\right)$ be defined by $\frac{1}{p(x)}-\frac{1}{q(x)}=\frac{\alpha}{n}$ and we suppose that the maximal operator is bounded on $L^{\left(q(\cdot) / q_{0}\right)^{\prime}}\left(\mathbb{R}^{n}\right)$, with $q_{0}$ such that $\frac{1}{p_{-}}-\frac{1}{q_{0}}=\frac{\alpha}{n}$.

If $p_{-}>1$ then there exist $C>0$ such that

$$
\left\|M_{\alpha} f\right\|_{q(\cdot)} \leq C\|f\|_{p(\cdot)} .
$$

If $p_{-}=1$ then, for all $\lambda>0$,

$$
\left\|\lambda \chi_{\left\{x \in \mathbb{R}^{n}:\left|M_{\alpha} f(x)\right|>\lambda\right\}}\right\|_{q(\cdot)} \leq C\|f\|_{p(\cdot)} .
$$

Proof. Let $q_{0}: \frac{1}{p_{-}}-\frac{1}{q_{0}}=\frac{\alpha}{n}$ and we suppose that $p_{-}>1$. Let $\tilde{q}(\cdot)=\frac{q(\cdot)}{q_{0}}$, we take a bounded function $f$ with compact support.

$$
\left\|M_{\alpha} f\right\|_{q(\cdot)}^{q_{0}}=\left\|\left(M_{\alpha} f\right)^{q_{0}}\right\|_{\tilde{q}(\cdot)} \leq C \sup _{\|h\|_{\tilde{q}^{\prime}(\cdot)}=1} \int\left(M_{\alpha} f\right)^{q_{0}}(x) h(x) d x,
$$


We define an iteration algorithm on $L^{\tilde{q}^{\prime}(\cdot)}\left(\mathbb{R}^{n}\right)$ by

$$
R h(x)=\sum_{k=0}^{\infty} \frac{M^{k} h(x)}{2^{k}\|M\|_{\tilde{q}^{\prime}(\cdot)}^{k}} .
$$

As in [2] it follows that

(i) $|h(x)| \leq R h(x)$ for all $x \in \mathbb{R}^{n}$.

(ii) $R$ is bounded on $L^{\tilde{q}^{\prime}(\cdot)}\left(\mathbb{R}^{n}\right)$ and $\|R h\|_{\tilde{q}^{\prime}(\cdot)} \leq 2\|h\|_{\tilde{q}^{\prime}(\cdot)}$

So

(iii) $R h \in \mathcal{A}_{1}$ and $[R h]_{\mathcal{A}_{1}} \leq 2\|M\|_{\tilde{q}^{\prime}(\cdot)}$

$$
\left\|M_{\alpha} f\right\|_{q(\cdot)}^{q_{0}} \leq C \sup _{\|h\|_{\tilde{q}^{\prime}(\cdot)}=1} \int\left(M_{\alpha} f\right)^{q_{0}}(x)\left(R h(x)^{\frac{1}{q_{0}}}\right)^{q_{0}} d x,
$$

since $R h \in \mathcal{A}_{1}$ then $R h^{\frac{1}{q_{0}}} \in \mathcal{A}_{1} \subset \mathcal{A}\left(p_{-}, q_{0}\right)$, and so, since $M_{\alpha}$ is bounded from $L^{p_{-}}\left(w^{p_{-}}\right)$in $L^{q_{0}}\left(w^{q_{0}}\right)$ for weights $w \in \mathcal{A}\left(p_{-}, q_{0}\right)$,

$$
\left\|M_{\alpha} f\right\|_{q(\cdot)}^{q_{0}} \leq C \sup _{\|h\|_{\tilde{q}^{\prime}(\cdot)}=1}\left(\int(f)^{p_{-}}(x)\left(R h(x)^{\frac{p_{-}}{q_{0}}}\right) d x\right)^{\frac{q_{0}}{p_{-}}}
$$

now we apply the Hölder's inequality with $\tilde{p}(\cdot)=\frac{p(\cdot)}{p_{-}}$,

$$
\begin{gathered}
\left\|M_{\alpha} f\right\|_{q(\cdot)}^{q_{0}} \leq C\left\|f^{p_{-}}\right\|_{\tilde{p}(\cdot)}^{\frac{q_{0}}{p-}} \sup _{\|h\|_{\tilde{q}^{\prime}(\cdot)}=1}\left\|R h^{\frac{p_{-}}{q_{0}}}\right\|_{\tilde{p}^{\prime}(\cdot)}^{\frac{q_{0}}{p_{-}}} \\
\leq C\|f\|_{p(\cdot)}^{q_{0}} .
\end{gathered}
$$

The rest of the proof follows as in the proof of Theorem 5.46 in [2], since $p_{+}<\infty$ and then the set of bounded functions with compact support is dense in $L^{p(\cdot)}\left(\mathbb{R}^{n}\right)$ (see Theorem 2.72 in [2]).

Lemma 2 Given $\Omega \subseteq \mathbb{R}^{n}$. If $p(\cdot) \in N_{\infty}(\Omega)$ and $p_{\infty}=\infty$ then $1 \in L^{p(\cdot)}(\Omega)$.

Proof. For $\lambda>1$ sufficiently large, by the $N_{\infty}$ - condition for $p$, and $\Omega_{+}=$ $\Omega \backslash \Omega_{\infty}$,

$$
\begin{gathered}
\int_{\Omega \backslash \Omega_{\infty}} \lambda^{-p(x)} d x=\int_{\Omega \backslash \Omega_{\infty}} e^{-p(x) \ln (\lambda)} d x \\
=\int_{\Omega \backslash \Omega_{\infty}} e^{-\Lambda_{\infty} p(x) \frac{\ln (\lambda)}{\Lambda_{\infty}}} d x \leq \int_{\Omega \backslash \Omega_{\infty}} e^{-\Lambda_{\infty} p(x)} d x<\infty .
\end{gathered}
$$

Proposition 3 Let $A$ be an invertible matrix $n \times n$.

a) If $p(\cdot) \in N_{\infty}\left(\mathbb{R}^{n}\right), 1 \leq p_{-} \leq p_{+}<\infty$ and $p(A x) \leq p(x)$ a.e.x $\in \mathbb{R}^{n}$, then there exists $c>0$ such that

$$
\left\|f \circ A^{-1}\right\|_{p(\cdot)} \leq c\|f\|_{p(\cdot)}
$$

for all $f \in L^{p(\cdot)}\left(\mathbb{R}^{n}\right)$.

b) If $p(A x)=p(x)$ a.e. $x \in \mathbb{R}^{n}$ then there exist $c>0$ such that

$$
\|f \circ A\|_{p(\cdot)} \leq c\|f\|_{p(\cdot)}
$$

for all $f \in L^{p(\cdot)}\left(\mathbb{R}^{n}\right)$. 
Proof. a) We assume that $f$ is bounded with compact support and $\|f\|_{p(\cdot)} \leq 1$. We will prove that,

$$
\left\|f \circ A^{-1}\right\|_{p(\cdot)} \leq c .
$$

We descompose $f=f_{1}+f_{2}$ where $f_{1}=f \chi_{\{x:|f(x)|>1\}}$ and $f_{2}=f \chi_{\{x:|f(x)| \leq 1\}}$,

$$
\left\|f \circ A^{-1}\right\|_{p(\cdot)} \leq\left\|f_{1} \circ A^{-1}\right\|_{p(\cdot)}+\left\|f_{2} \circ A^{-1}\right\|_{p(\cdot)} .
$$

We define $E=\left\{x: p(x) \geq p_{\infty}\right\}$ and $F=\left\{x: p(x)<p_{\infty}\right\}$. We estimate $\left\|f_{2} \circ A^{-1}\right\|_{p(\cdot)}$,

$$
\left\|f_{2} \circ A^{-1}\right\|_{L^{p(\cdot)}} \leq\left\|f_{2} \circ A^{-1}\right\|_{L^{p(\cdot)}(E)}+\left\|f_{2} \circ A^{-1}\right\|_{L^{p(\cdot)}(F)} .
$$

Since $f_{2}$ is bounded and with compact support, $f_{2} \in L^{p_{\infty}}\left(\mathbb{R}^{n}\right)$ and so

$$
f_{2} \circ A^{-1} \in L^{p_{\infty}}\left(\mathbb{R}^{n}\right)
$$

Lemma 3.28 in [2], with $g=f_{2} \circ A^{-1}, t(\cdot)=p_{\infty}, u(\cdot)=p(\cdot)$ implies that, if $\left\|f_{2} \circ A^{-1}\right\|_{L^{p_{\infty}(E)}}<1$ then

$$
\left\|f_{2} \circ A^{-1}\right\|_{L^{p(\cdot)(E)}} \leq\left\|f_{2} \circ A^{-1}\right\|_{L^{p \infty}(E)}+1<2 .
$$

and if $\left\|f_{2} \circ A^{-1}\right\|_{L^{p \infty}(E)} \geq 1$ then

$$
\begin{gathered}
\left\|f_{2} \circ A^{-1}\right\|_{L^{p(\cdot)}(E)} \leq 2\left\|f_{2} \circ A^{-1}\right\|_{L^{p \infty}(E)} \leq 2 \operatorname{det}(A)\left\|f_{2}\right\|_{L^{p_{\infty}\left(\mathbb{R}^{n}\right)}} \\
\leq 2 \operatorname{det}(A)\left[\left\|f_{2}\right\|_{L^{p_{\infty}(E)}}+\left\|f_{2}\right\|_{L^{p_{\infty}(F)}}\right] .
\end{gathered}
$$

To estimate $\left\|f_{2}\right\|_{L^{p_{\infty}(E)}}$, by the definition of $E$, we define the defect exponent $r(\cdot) \in \mathcal{P}(E)$ by

$$
\frac{1}{p_{\infty}}=\frac{1}{p(x)}+\frac{1}{r(x)}
$$

By the generalizated Hölder's inequality, Corollary 2.28 in [2],

$$
\left\|f_{2}\right\|_{L^{p \infty}(E)} \leq K\|1\|_{L^{r(\cdot)}(E)}\left\|f_{2}\right\|_{L^{p(\cdot)}(E)} \leq K\|1\|_{L^{r(\cdot)}(E)}<\infty .
$$

The last inequality follows since $r(\cdot) \in N_{\infty}, r_{\infty}=\infty$ and so Lemma 2 implies $1 \in L^{r(\cdot)}(E)$.

To estimate $\left\|f_{2}\right\|_{L^{p_{\infty}(F)}}$, we apply Lemma 3.28 in [2], with $g=f_{2} \in L^{p(\cdot)}(F)$, $t(\cdot)=p(\cdot), u(\cdot)=p_{\infty}$. Since $\left\|f_{2}\right\|_{L^{p(\cdot)}(F)} \leq 1$,

$$
\left\|f_{2}\right\|_{L^{p} \infty(F)} \leq\left\|f_{2}\right\|_{L^{p(\cdot)}\left(\mathbb{R}^{n}\right)}+1 \leq 2 .
$$

Combining the above estimates we get

$$
\left\|f_{2} \circ A^{-1}\right\|_{L^{p(\cdot)}(E)} \leq C\left(K\|1\|_{L^{r(\cdot)}(E)}+2\right)<\infty .
$$

Now, in a similar way, we estimate $\left\|f_{2} \circ A^{-1}\right\|_{L^{p(\cdot)}(F)}$. We define the defect exponent $s(\cdot) \in \mathcal{P}(F)$ by

$$
\frac{1}{p(x)}=\frac{1}{p_{\infty}}+\frac{1}{s(x)}
$$


By the generalized Hölder's inequality, Corollary 2.28 in [2],

$$
\left\|f_{2} \circ A\right\|_{L^{p(\cdot)}(F)}^{-1} \leq K\|1\|_{L^{s(\cdot)}(F)}\left\|f_{2} \circ A^{-1}\right\|_{L^{p \infty}(F)} .
$$

Since $s(\cdot) \in N_{\infty}$ and $s_{\infty}=\infty$, by Lemma 2 we have that $1 \in L^{s(\cdot)}(F)$. Further, we can now argue as we did above to get

$$
\begin{gathered}
\left\|f_{2} \circ A^{-1}\right\|_{L^{p_{\infty}(F)}} \leq\left\|f_{2} \circ A^{-1}\right\|_{L^{p_{\infty}\left(\mathbb{R}^{n}\right)}} \leq \operatorname{det}(A)\left\|f_{2}\right\|_{L^{p \infty}\left(\mathbb{R}^{n}\right)} \\
\leq \operatorname{det}(A)\left[\left\|f_{2}\right\|_{L^{p_{\infty}(E)}}+\left\|f_{2}\right\|_{L^{p_{\infty}(F)}}\right]<\infty .
\end{gathered}
$$

We now estimate $\left\|f_{1} \circ A^{-1}\right\|_{p(\cdot)}$. Since $p_{+}<\infty$ it's enough to prove that there exits $c>0$ such that $\rho_{p(\cdot)}\left(f_{1} \circ A^{-1}\right) \leq c$. Since $p(A x) \leq p(x)$ a.e. $x \in \mathbb{R}^{n}$ and again from Corollary 2.22 in [2],

$$
\int f_{1}\left(A^{-1} x\right)^{p(x)} d x=\operatorname{det}(A) \int f_{1}(x)^{p(A x)} d x \leq \operatorname{det}(A) \rho_{p(\cdot)}\left(f_{1}\right) \leq c\left\|f_{1}\right\|_{p(\cdot)}<c .
$$

For general $f \in L^{p(.)}\left(\mathbb{R}^{n}\right)$, we apply Theorem 2.59 in [2]. For $k \in \mathbb{N}$, we define $f^{k}(x)=|f| \chi_{\{x:|x| \leq k,|f(x)| \leq k\}}, f^{k}(x)$ increases to $|f(x)|$ pointwise almost everyhere and so $\left\|f^{k}\right\|_{p(.)} \rightarrow\|f\|_{p(.)}$ and also $\left\|f^{k} \circ A^{-1}\right\|_{p(.)} \rightarrow\left\|f \circ A^{-1}\right\|_{p(.)}$. Since each $f^{k}$ is a bounded function with compact support and $\left\|\frac{f_{k}}{\left\|f_{k}\right\|_{p(.)}}\right\| \leq 1$, we have proved that there exists a constant $c>0$ such that $\left\|\frac{f_{k} \circ A^{-1}}{\left\|f_{k}\right\|_{p(.)}}\right\|_{p(.)} \leq c$, so

$$
\left\|\frac{f \circ A^{-1}}{\|f\|_{p(.)}}\right\|_{p(.)}=\lim _{k \rightarrow \infty}\left\|\frac{f_{k} \circ A^{-1}}{\|f\|_{p(.)}}\right\|_{p(.)} \leq \lim _{k \rightarrow \infty}\left\|\frac{f_{k} \circ A^{-1}}{\left\|f_{k}\right\|_{p(.)}}\right\|_{p(.)} \leq c,
$$

and then

$$
\left\|f \circ A^{-1}\right\|_{p(.)} \leq c\|f\|_{p(.)} .
$$

b) Let $f \in L^{p(\cdot)}\left(\mathbb{R}^{n}\right)$. We have that

$$
\|f \circ A\|_{p(\cdot)}=\inf \left\{\lambda>0: \int_{\mathbb{R}^{n}}\left(\frac{f(A x)}{\lambda}\right)^{p(x)} d x \leq 1\right\} .
$$

By a change of variable and using the hypothesis on the exponent,

$$
\int_{\mathbb{R}^{n}}\left(\frac{f(A x)}{\lambda}\right)^{p(x)} d x=\left|\operatorname{det}\left(A^{-1}\right)\right| \int_{\mathbb{R}^{n}}\left(\frac{f(y)}{\lambda}\right)^{p(y)} d y,
$$

Let $D=\left|\operatorname{det}\left(A^{-1}\right)\right|$, then we have two cases: If $D \leq 1$,

$$
\|f \circ A\|_{p(\cdot)} \leq\|f\|_{p(\cdot)} .
$$

If $D>1$, then from (4) it is follows that

$$
D \int_{\mathbb{R}^{n}}\left(\frac{f(y)}{\lambda}\right)^{p(y)} d y=\int_{\mathbb{R}^{n}}\left(\frac{f(y)}{\lambda C^{\frac{1}{p(y)}}}\right)^{p(y)} d y,
$$


where $C=\frac{1}{D}$. So,

$$
\leq \int_{\mathbb{R}^{n}}\left(\frac{f(y)}{\lambda C^{\frac{1}{p_{-}}}}\right)^{p(y)} d y
$$

That is,

$$
\int_{\mathbb{R}^{n}}\left(\frac{f(A x)}{\lambda}\right)^{p(x)} d x \leq \int_{\mathbb{R}^{n}}\left(\frac{f(x)}{\lambda C^{\frac{1}{p_{-}}}}\right)^{p(x)} d x .
$$

From this last inequality it follows that

$$
\|f \circ A\|_{p(\cdot)} \leq D^{\frac{1}{p_{-}}}\|f\|_{p(\cdot)} .
$$

Theorem 4 Let $0 \leq \alpha<n$ and let $T_{\alpha}$ be the integral operator given by (3). Let $m \in \mathbb{N}$ (or $m \in \mathbb{N} \backslash\{1\}$ for $\alpha=0$ ), let $A_{1}, \ldots, A_{m}$ be invertible matrices such that $A_{i}-A_{j}$ is invertible for $i \neq j, 1 \leq i, j \leq m$ and the functions $\Omega_{i}$ satisfy the hypothesis (H1) and (H2). Let $s \geq 1$ be defined by $\frac{1}{p_{1}}+\ldots+\frac{1}{p_{m}}+\frac{1}{s}=1$, let $p(\cdot) \in \mathcal{P}\left(\mathbb{R}^{n}\right)$ be such that $1 \leq s \leq p_{-} \leq p_{+}<\frac{n}{\alpha}$ and such that $p\left(A_{i} x\right) \leq p(x)$ a.e. $x \in \mathbb{R}^{n}$ and let $q(\cdot) \in \mathcal{P}\left(\mathbb{R}^{n}\right)$ be defined by $\frac{1}{p(\cdot)}-\frac{1}{q(\cdot)}=\frac{\alpha}{n}$. If $\frac{q(\cdot)}{s} \in$ $N_{\infty}\left(\mathbb{R}^{n}\right) \cap K_{0}\left(\mathbb{R}^{n}\right)$ then,

a) there exist $C>0$ such that

$$
\left\|\lambda \chi_{\left\{x: T_{\alpha} f(x)>\lambda\right\}}\right\|_{q(\cdot)} \leq C\|f\|_{p(\cdot)}
$$

for all $\lambda>0, f \in L_{c}^{\infty}\left(\mathbb{R}^{n}\right)$.

b) If $p_{-}>s$ then $T_{\alpha}$ extends to a bounded operator from $L^{p(\cdot)}\left(\mathbb{R}^{n}\right)$ into $L^{q(\cdot)}\left(\mathbb{R}^{n}\right)$.

Proof. a) In [6] the authors prove that, for $f \in L_{c}^{\infty}\left(\mathbb{R}^{n}\right)$,

$$
M^{\#}\left(T_{\alpha} f\right)(x) \leq c \sum_{i=1}^{m} M_{\alpha, s} f\left(A_{i}^{-1} x\right),
$$

a.e. $x \in \mathbb{R}^{n}$, where $M_{\alpha, s}$ is defined in 1. Since $\frac{q(\cdot)}{s} \in N_{\infty}\left(\mathbb{R}^{n}\right) \cap K_{0}\left(\mathbb{R}^{n}\right)$ then $q^{\prime}(\cdot) \in N_{\infty}\left(\mathbb{R}^{n}\right) \cap K_{0}\left(\mathbb{R}^{n}\right)$. Indeed is easy to check that if $p(\cdot) \in N_{\infty}\left(\mathbb{R}^{n}\right)$ then $\alpha p(\cdot) \in N_{\infty}\left(\mathbb{R}^{n}\right)$ for all $\alpha \geq 1$. So $s \frac{q(\cdot)}{s}=q(\cdot) \in N_{\infty}\left(\mathbb{R}^{n}\right)$. Remark 4.6 in [2] implies $q^{\prime}(\cdot) \in N_{\infty}\left(\mathbb{R}^{n}\right)$. Since $\frac{q(\cdot)}{s} \in N_{\infty}\left(\mathbb{R}^{n}\right) \cap K_{0}\left(\mathbb{R}^{n}\right)$ then by Theorem 4.52 in [2] the maximal operator it is bounded on $L^{\frac{q(\cdot)}{s}}\left(\mathbb{R}^{n}\right)$. So, by Theorem 4.37 in [2], it is bounded on $L^{q(\cdot)}\left(\mathbb{R}^{n}\right)$. Also by Corollary 4.64 in [2] it is bounded on $L^{q^{\prime}(\cdot)}\left(\mathbb{R}^{n}\right)$. By Corollary 4.50 in $[2], q^{\prime}(\cdot) \in K_{0}\left(\mathbb{R}^{n}\right)$. And so $q^{\prime}(\cdot) \in N_{\infty}\left(\mathbb{R}^{n}\right) \cap K_{0}\left(\mathbb{R}^{n}\right)$.

Let $\lambda>0$ and $f \in L_{c}^{\infty}\left(\mathbb{R}^{n}\right)$. Since $q^{\prime}(\cdot) \in N_{\infty}\left(\mathbb{R}^{n}\right) \cap K_{0}\left(\mathbb{R}^{n}\right)$, again Theorem 4.52 in [2] implies that the maximal operator is bounded on $L^{q^{\prime}(\cdot)}\left(\mathbb{R}^{n}\right)$, so from Theorem 5.54 in [2] and (5),

$$
\begin{gathered}
\left\|\lambda \chi_{\left\{x: T_{\alpha} f(x)>\lambda\right\}}\right\|_{q(\cdot)} \leq C \sup _{\lambda>0}\left\|\lambda \chi_{\left\{x: M \#\left(T_{\alpha} f\right)(x)>\lambda\right\}}\right\|_{q(\cdot)} \\
\leq C \sup _{\lambda>0}\left\|\lambda \chi_{\left\{x: \sum_{i=1}^{m} M_{\alpha, s} f\left(A_{i}^{-1} x\right)>\frac{\lambda}{c}\right\}}\right\|_{q(\cdot)} \leq C \sup _{\lambda>0}\left\|\lambda \sum_{i=1}^{m} \chi_{\left\{x: M_{\alpha, s} f\left(A_{i}^{-1} x\right)>\frac{\lambda}{c m}\right\}}\right\|_{q(\cdot)}
\end{gathered}
$$




$$
\leq C \sup _{\lambda>0} \sum_{i=1}^{m}\left\|\lambda \chi_{\left\{x: M_{\alpha, s} f\left(A_{i}^{-1} x\right)>\frac{\lambda}{c m}\right\}}\right\|_{q(\cdot)} \leq C \sup _{\lambda>0} \sum_{i=1}^{m}\left\|\lambda \chi_{\left\{x: M_{\alpha . s}|f|^{s}\left(A_{i}^{-1} x\right)>\left(\frac{\lambda}{c m}\right)^{s}\right\}}\right\|_{q(\cdot)}
$$

So by Proposition 2.18 in [2] and by Theorem 2.34 in [2]

$$
\begin{gathered}
\left\|\lambda \chi_{\left\{x: T_{\alpha} f(x)>\lambda\right\}}\right\|_{q(\cdot)} \leq C \sup _{\lambda>0} \sum_{i=1}^{m}\left\|\lambda^{s} \chi_{\left\{x: M_{\alpha \cdot s}|f|^{s}\left(A_{i}^{-1} x\right)>\left(\frac{\lambda}{c m}\right)^{s}\right\}}\right\|_{\frac{(q \cdot)}{s}}^{\frac{1}{s}} \\
\leq C \sup _{\lambda>0} \sum_{i=1}^{m}\left\|\left(\frac{\lambda}{c m}\right)^{s} \chi_{\left\{x: M_{\alpha \cdot s}|f|^{s}\left(A_{i}^{-1} x\right)>\left(\frac{\lambda}{c m}\right)^{s}\right\}}\right\|_{\frac{q(\cdot)}{s}}^{\frac{1}{s}} \\
\leq C \sup _{\lambda>0} \sum_{i=1}^{m}\left[\sup _{\|h\|_{\left(\frac{q(\cdot)}{s}\right)^{\prime}}=1} \int_{\left\{x: M_{\alpha \cdot s}|f|^{s}\left(A_{i}^{-1} x\right)>\left(\frac{\lambda}{c m}\right)^{s}\right\}}\left(\frac{\lambda}{c m}\right)^{s} h(x) d x\right]^{\frac{1}{s}} \\
=C \sup _{\lambda>0} \sum_{i=1}^{m}\left[\sup _{\|h\|_{\left(\frac{q(\cdot)}{s}\right)^{\prime}}} \int_{A_{i}^{-1}\left\{x: M_{\alpha \cdot s}|f|^{s}\left(A_{i}^{-1} x\right)>\left(\frac{\lambda}{c m}\right)^{s}\right\}}\left(\frac{\lambda}{c m}\right)^{s} h\left(A_{i} y\right) d y\right]^{\frac{1}{s}} \\
\leq C \sup _{\lambda>0} \sum_{i=1}^{m}\left[\sup _{\|h\|} \int_{\left(\frac{q(\cdot)}{s}\right)^{\prime}=1} \int_{\left\{y: M_{\alpha \cdot s}|f|^{s}(y)>\left(\frac{\lambda}{c m}\right)^{s}\right\}}\left(\frac{\lambda}{c m}\right)^{s} h\left(A_{i} y\right) d y\right]^{\frac{1}{s}}
\end{gathered}
$$

We first apply the Hölder's inequality (Theorem 2.26 in [2]). Then we use $\left(\frac{q(\cdot)}{s}\right)^{\prime} \in N_{\infty}\left(\mathbb{R}^{n}\right)$, Proposition 3, Lemma 1 and Proposition 2.18 in [2] to obtain

$$
\left\|\lambda \chi_{\left\{x: T_{\alpha} f(x)>\lambda\right\}}\right\|_{q(\cdot)}
$$

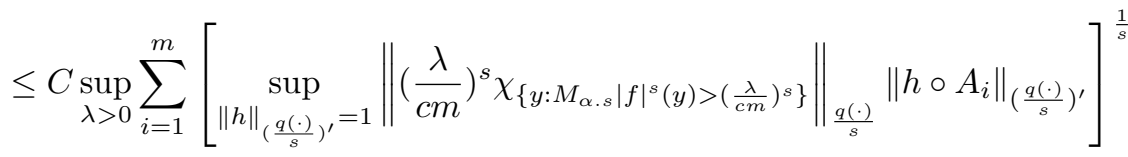

$$
\begin{aligned}
& \leq C \sup _{\lambda>0} \sum_{i=1}^{m}\left[\sup _{\|h\| \|_{\left(\frac{q(\cdot)}{s}\right)^{\prime}}=1}\left\|\left(\frac{\lambda}{c m}\right)^{s} \chi_{\left\{y: M_{\alpha \cdot s}|f|^{s}(y)>\left(\frac{\lambda}{c m}\right)^{s}\right\}}\right\|_{\frac{q(\cdot)}{s}}\|h\|_{\left(\frac{q(\cdot)}{s}\right)^{\prime}}\right]^{\frac{1}{s}} \\
& \leq C \sup _{\lambda>0}\left\|\left(\frac{\lambda}{c m}\right)^{s} \chi_{\left\{y: M_{\alpha . s}|f|^{s}(y)>\left(\frac{\lambda}{c m}\right)^{s}\right\}}\right\|_{\frac{q(\cdot)}{s}}^{\frac{1}{s}} \leq C\left\||f|^{s}\right\|_{\frac{p(\cdot)}{s}}^{\frac{1}{s}}=C\|f\|_{p(\cdot)} \text {. }
\end{aligned}
$$

b) Let $f \in L_{c}^{\infty}\left(\mathbb{R}^{n}\right)$. By Theorem 5.54 in $[2]$, since $q^{\prime}(\cdot) \in N_{\infty}\left(\mathbb{R}^{n}\right) \cap K_{0}\left(\mathbb{R}^{n}\right)$,

$$
\left\|T_{\alpha} f\right\|_{q(\cdot)} \leq C\left\|M^{\#}\left|T_{\alpha} f\right|\right\|_{q(\cdot)}
$$

Now we use (5) and since $q(\cdot) \in N_{\infty}\left(\mathbb{R}^{n}\right)$, by the Proposition 3

$$
\leq \sum_{i=1}^{m}\left\|M_{\alpha, s} f\left(A_{i}^{-1} \cdot\right)\right\|_{q(\cdot)} \leq C \sum_{i=1}^{m}\left\|M_{\alpha, s} f\right\|_{q(\cdot)}=C m\left\|\left(M_{\alpha . s}|f|^{s}\right)^{1 / s}\right\|_{q(\cdot)}
$$


By the Proposition 2.18 in [2], Lemma 1 and Proposition 2.18 in [2]

$$
=C m\left\|\left(M_{\alpha . s}|f|^{s}\right)\right\|_{\frac{q(\cdot)}{s}}^{\frac{1}{s}} \leq C\left\||f|^{s}\right\|_{\frac{p(\cdot)}{s}}^{\frac{1}{s}}=C\|f\|_{p(\cdot)} .
$$

Now $b$ ) follows since $L_{c}^{\infty}\left(\mathbb{R}^{n}\right)$ is dense in $L^{p(\cdot)}\left(\mathbb{R}^{n}\right)$.

Theorem 5 Let $0 \leq \alpha<n$ and let $T_{\alpha}$ be the integral operator given by (3). Let $m \in \mathbb{N}$ (or $m \in \mathbb{N} \backslash\{1\}$ for $\alpha=0$ ). Let $A_{1}, \ldots, A_{m}$ be invertible matrices such that $A_{i}-A_{j}$ is invertible for $i \neq j, 1 \leq i, j \leq m$ and the functions $\Omega_{i}$ satisfy the hypothesis $\left(H_{1}\right)$ and $\left(H_{2}\right)$. Let $s \geq 1$ be defined by $\frac{1}{p_{1}}+\ldots+\frac{1}{p_{m}}+\frac{1}{s}=1$, let $p(\cdot) \in \mathcal{P}\left(\mathbb{R}^{n}\right)$ be such that $1 \leq s \leq p_{-} \leq p_{+}<\frac{n}{\alpha}$ and such that $p\left(A_{i} x\right)=$ $p(x)$ a.e. $x \in \mathbb{R}^{n}$ and let $q(\cdot) \in \mathcal{P}\left(\mathbb{R}^{n}\right)$ be defined by $\frac{1}{p(\cdot)}-\frac{1}{q(\cdot)}=\frac{\alpha}{n}$. If the maximal operator is bounded on $L^{q^{\prime}(\cdot)}\left(\mathbb{R}^{n}\right)$ then,

a) there exist $c>0$ such that

$$
\left\|\lambda \chi_{\left\{x: T_{\alpha} f(x)>\lambda\right\}}\right\|_{q(\cdot)} \leq c\|f\|_{p(\cdot)}
$$

for all $\lambda>0, f \in L_{c}^{\infty}\left(\mathbb{R}^{n}\right)$.

b) If $p_{-}>s$ then $T_{\alpha}$ extendes to a bounded operator from $L^{p(\cdot)}\left(\mathbb{R}^{n}\right)$ into $L^{q(\cdot)}\left(\mathbb{R}^{n}\right)$.

Proof. a) Let $\lambda>0$ and $f \in L_{c,}^{\infty}\left(\mathbb{R}^{n}\right)$. By Theorem 5.54 in [2], since the maximal operator is bounded on $L^{q^{\prime}(\cdot)}\left(\mathbb{R}^{n}\right)$,

$$
\left\|\lambda \chi_{\left\{x: T_{\alpha} f(x)>\lambda\right\}}\right\|_{q(\cdot)} \leq C\left\|\lambda \chi_{\left\{x: M \#\left(T_{\alpha} f\right)(x)>\lambda\right\}}\right\|_{q(\cdot)} .
$$

Now, by (5), as in the proof of the previous theorem and since $\left(\frac{q\left(A_{i} x\right)}{s}\right)^{\prime}=$ $\left(\frac{q(x)}{s}\right)^{\prime}$, by Proposition 3, Lemma 1 and Proposition 2.18 in [2], we have that

$$
\begin{aligned}
& \left\|\lambda \chi_{\left\{x: T_{\alpha} f(x)>\lambda\right\}}\right\|_{q(\cdot)} \leq
\end{aligned}
$$

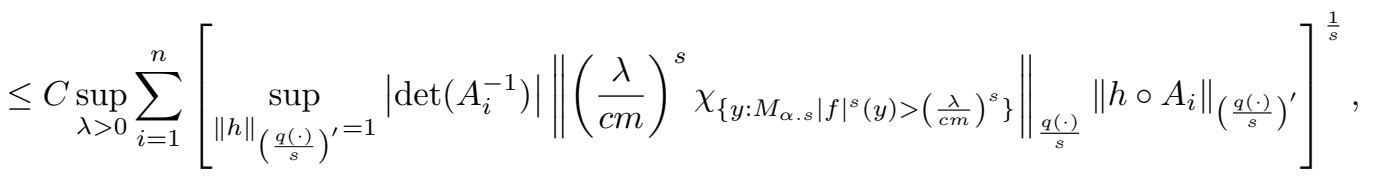

$$
\begin{aligned}
& \leq C \sup _{\lambda>0} \sum_{i=1}^{n}\left[\sup _{\|h\|_{\left(\frac{q(\cdot)}{s}\right)^{\prime}=1}}\left|\operatorname{det}\left(A_{i}^{-1}\right)\right|\left\|\left(\frac{\lambda}{c m}\right)^{s} \chi_{\left\{y: M_{\alpha . s}|f|^{s}(y)>\left(\frac{\lambda}{c m}\right)^{s}\right\}}\right\| \frac{q(\cdot)}{s}\|h\|_{\left(\frac{q(\cdot)}{s}\right)^{\prime}}\right]^{\frac{1}{s}} \\
& \leq C \sup _{\lambda>0}\left\|\left(\frac{\lambda}{c m}\right)^{s} \chi_{\left\{y: M_{\alpha \cdot s}|f|^{s}(y)>\left(\frac{\lambda}{c m}\right)^{s}\right\}}\right\|_{\frac{q(\cdot)}{s}} \leq C\left\||f|^{s}\right\|_{\frac{p(\cdot)}{s}}^{\frac{1}{s}}=C\|f\|_{p(\cdot)} .
\end{aligned}
$$

b) We suppose that $s<p_{-}$. Let $f \in L_{c}^{\infty}\left(\mathbb{R}^{n}\right)$. By Theorem 5.54 in [2], since the maximal operator is bounded on $L^{q^{\prime}(\cdot)}\left(\mathbb{R}^{n}\right)$,

$$
\left\|T_{\alpha} f\right\|_{q(\cdot)} \leq C\left\|M^{\#}\left(T_{\alpha} f\right)\right\|_{q(\cdot)}
$$

by (5) and since $q\left(A_{i} x\right)=q(x)$, by Proposition 3 ,

$$
\left\|T_{\alpha} f\right\|_{q(\cdot)} \leq C \sum_{i=1}^{m}\left\|M_{\alpha, s} f\left(A_{i}^{-1} \cdot\right)\right\|_{q(\cdot)} \leq C \sum_{i=1}^{m}\left\|M_{\alpha, s} f\right\|_{q(\cdot)} \leq C\|f\|_{p(\cdot)} .
$$

where the last inequality follows as in the proof of the previous theorem. 


\section{References}

[1] Capone C., Cruz Uribe D., Fiorenza A. The fractional maximal operator and fractional integrals on variable $L^{p}$ spaces, Rev. Mat. Ibeoamericana, 2, (2007), 743-770.

[2] Cruz Uribe D., Fiorenza A., Variable Lebesgue Spaces, Foundations and Harmonic Analysis, (Birkhäuser, 2013).

[3] Cruz Uribe D., Fiorenza A., Neugebauer C. J., The maximal function on variable $L^{p}$ spaces, Ann. Acad. Sci. Fenn. Math., 28, (2003), 223-238.

[4] Diening L., Harjulehto P., Hästö P., Ruzicka M., Lebesgue and Sovoleb Spaces with variable exponents, Lecture Notes in Mathematics 2017, (SpringerVerlag Berlin Heidelberg 2011).

[5] Muckenhoupt B., Wheeden R. L., Weighted norm inequalities for fractional integrals, Trans. Amer. Math. Soc.,192, (1974), 261-274.

[6] Riveros M. S., Urciuolo M., Weighted inequalities for some integral operators with Rough kernels, Acta Mathematica Sinica, 29, (2013), 449-460.

[7] Rocha P., Urciuolo M., About integral operators of fractional type on variable $L^{p}$ spaces, Georgian Math. J., 20, (2013), 805-816.

[8] Urciuolo M., Vallejos L., $L^{p(.)}-L^{q(.)}$ boundedness of some integral operators obtained by extrapolation techniques, Georgian Math. J., DOI: https://doi.org/10.1515/gmj-2018-0066, (2018).

[9] Urciuolo M., Vallejos L., A generalization of the boundedness of certain integral operators in variable Lebesgue spaces, Journal of Mathematical Inequalities, JMI-3138, to appear.

- Marta Urciuolo, FAMAF, UNIVERSIDAD NACIONAL DE CORDOBA, CIEM, CONICET, Ciudad Universitaria, 5000 Córdoba, Argentina.

E-mail adress: urciuolo@gmail.com

- Lucas Vallejos, FAMAF, UNIVERSIDAD NACIONAL DE CORDOBA, CIEM, CONICET, Ciudad Universitaria, 5000 Córdoba, Argentina.

E-mail adress: lvallejos@famaf.unc.edu.ar 